\title{
Velocity measurements of dilute suspensions over and through various porous media models
}

\author{
E. A. Haffner ${ }^{1}$, T. Wilkie ${ }^{1}$, J. E. Higham ${ }^{2}$, P. Mirbod ${ }^{* 1}$ \\ ${ }^{1}$ University of Illinois at Chicago, Department of Mechanical and Industrial Engineering, Chicago, USA \\ ${ }^{2}$ University of Liverpool, School of Environmental Sciences, Liverpool, UK \\ *pmirbod@uic.edu
}

\begin{abstract}
This study is focused on the motion of a dilute suspension containing rigid, spherical, non-Brownian, noncolloidal particles flowing over and through porous media models. The flow is confined to very low Reynolds numbers. To examine the velocity distribution particle image velocimetry (PIV) was applied in conjunction with refractive index matching (RIM) techniques. This study is the first of its kind analyzing the interaction between two common engineering systems: suspension fluid and porous media.
\end{abstract}

\section{Introduction}

Porous media has been a prevalent structure in both the natural world as well as many manufactured systems. Fluid motion over sediment beds Goharzadeh et al. (2005) and coral reefs/ submerged vegetation Ghisalberti and Nepf (2009) have been investigated as natural forms of porous media. Other studies have focused on using optical experimental techniques to investigate the properties at the interface of a porous media bounded on top by a free flow region. Specifically, PIV experiments were developed to identify the effect of the porous media characteristics and different porous media configurations on the overall flow structure and the properties at the interface, Arthur et al. (2009); Agelinchaab et al. (2006). These studies are limited to pure Newtonian fluid over and through porous media.

Much like porous media, suspension flows are observed in natural systems, so they have been commonly utilized in engineering structures. Because of this, optical experimental techniques were developed coupled with RIM techniques to measure the velocity and concentration for suspension flow through various geometries. These PIV experiments have been developed to study various phenomenon within the velocity profile for different types of suspensions, Jesinghausen et al. (2016); Medhi et al. (2011).

By examining previous literature, it was clear that there was a fundamental gap in knowledge that describes how these two systems would interact to one another. These types of systems have been observed in pharmaceuticals, oil mining, and slurry transport. It is important to better understand how the properties of both systems would affect the flow structure. This study using PIV to study how the velocity and interaction properties are effected by the porous media characteristics and the dilute suspension concentration.

The suspension particles were monodispersed polymethyl methacrylate (PMMA) with an average size of $82 \mu \mathrm{m}$ (Cospheric, LLC). The Newtonian solvent was created based off of a solution presented by Lyon and Leal (1998a, 199b) Lyon and Leal (1998a b). Multiple dimensionless parameters such as particle Reynolds numbers, Stokes number, and Pélect number were all checked to ensure the motion of the suspension particles was solely dependent on the fluid motion. The porous media was made up of rigid rods in a unit square and was created by a 3D printer. This was done so that we could control the various properties of the porous media. There were three dimensionless height parameters, $\delta=h_{p} / L$ tested, $\delta=4.54,1.37,0.74$ and three permeability parameters $\sigma=L / \sqrt{K}$ tested, $\sigma=2.2,3.46,6$.09. In these relationships $h_{p}$ is the porous media thickness, $L$ is half the free flow height, and $K$ is the permeability. 

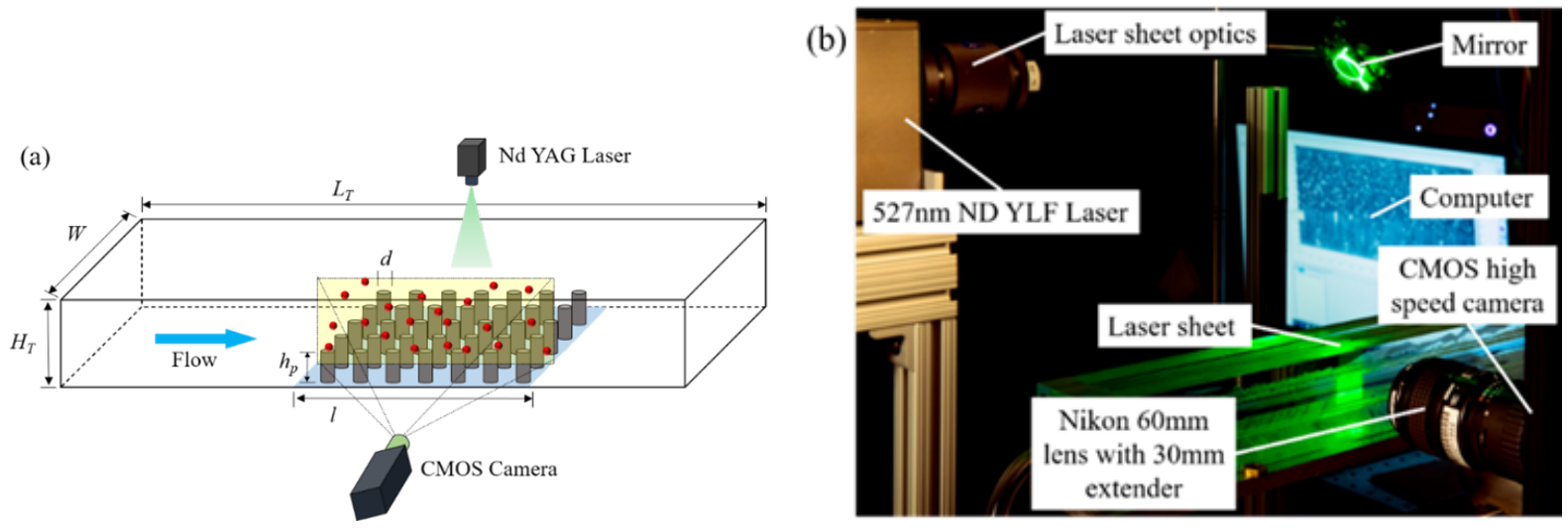

Figure 1: (a) Schematic of the experimental setup, and (b) an image of the experiments in Mirbod's Lab.

\section{Results}

The velocity fields were extracted from two data collection planes, one plane within the rods of the porous media, $P_{1}$, and one plane placed in the middle on top of the rods, $P_{2}$. These two planes were area averaged together to get the full one-dimensional velocity profile for the flow through the porous media, Arthur et al. (2009). These velocity profiles for the different $\delta$ values are shown in Fig. 2. In each figure the three different permeability parameters are shown at each constant height ratio. In these figures, as the permeability parameter decreases the lower the velocity is within the porous media. For the largest height ratio, Fig. 2(c), there is an observable effect on the velocity profile for the highest permeability parameter. For the other two height ratios there is not much of an effect at $\sigma=6.09$.

Not only are the velocity profiles examined by also the properties at the interface between the free flow region and the top of the porous media. The slip velocity at the interface, $U_{s}=\left|U_{\text {ave }}\right|_{(y / L=0)}$, Lauga and Stone (2003), is normalized using two different methods. The first is using the maximum velocity in the free flow region, which is shown in Fig. 3(a) for the three different delta values. The other method, known as the dimensionless slip parameter, uses local parameters such as the shear rate $\dot{\gamma}=|d u / d y|_{y=0}$ and the permeability. This parameter better describes the properties at the interface and is known and the dimensionless slip parameter. This is shown in Fig. 2(b) for both data collection planes and the averaged profile. Fig. 3(c) shows the slip length which is length between the actual location of the velocity profile and the location where the parabolic velocity profile would be if it were extrapolated to the interface. Mathematically, this is defined as $l_{\text {slip }}=U_{s} /|d u / d y|_{y=0}$. Fig 3 (a) and Fig, 3 (c) shows that there is a negative trend as $\sigma$ increases for all $\delta$. Fig. 3 (b) shows that as $\delta$ increases so does the dimensionless slip parameters except for $\delta=4.54$ cases which show a negative trend.

\section{Acknowledgements}

We would like to thank NSF CBET Award \#1854376 for funding this work. 

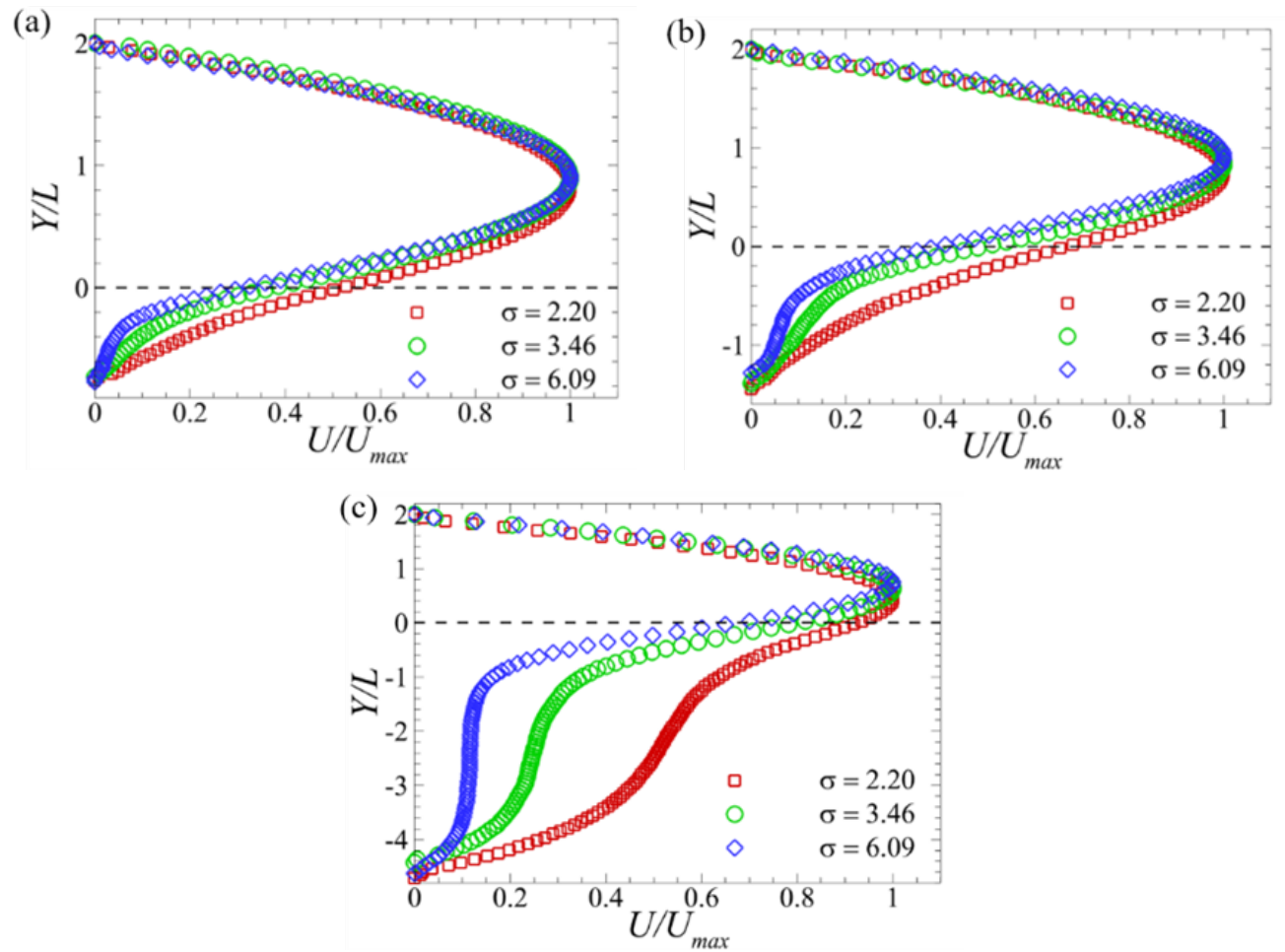

Figure 2: The velocity profiles for various permeability parameters $\sigma$ and different depth ratio (a) $\delta=0.74$, (b) $\delta=1.37$, and (c) $\delta=4.54$. 

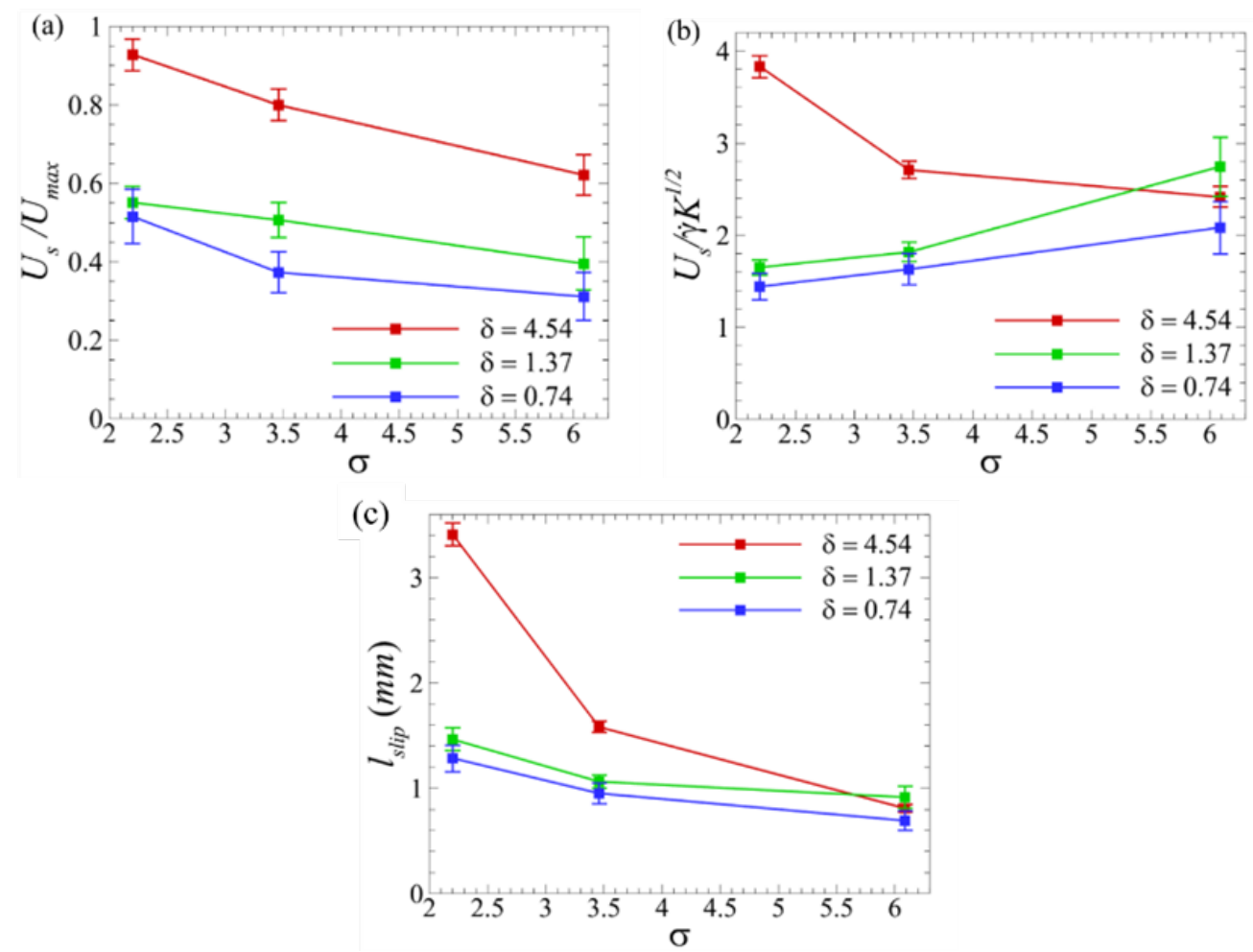

Figure 3: (a) The ratio of the slip velocity to the maximum velocity for the various test cases. (b) The dimensionless slip parameter for all test cases and the (d) slip length evaluated at the interface. The error bars were calculated using the velocity uncertainties and methods outlined by Sciacchitano and Wieneke (2016) and Coleman and Steele (1995) 


\section{References}

Agelinchaab M, Tachie MF, and Ruth DW (2006) Velocity measurement of flow through a model threedimensional porous medium. Physics of Fluids 18:017105-11

Arthur JK, Ruth DW, and Tachie MF (2009) Piv measurements of flow through a model porous medium with varying boundary conditions. Journal of Fluid Mechanics 629:343-374

Coleman HW and Steele WG (1995) Engineering application of experimental uncertainty analysis. AIAA Journal 33:1888-1896

Ghisalberti M and Nepf H (2009) Shallow flows over a permeable medium: The hydrodynamics of submerged aquatic canopies. Transport in Porous Media 78:309-326

Goharzadeh A, Khalili A, and Jørgensen BB (2005) Transition layer thickness at a fluid-porous interface. Physics of Fluids 17:057102-10

Jesinghausen S, Weiffen R, and Schmid HJ (2016) Direct measurement of wall slip and slip layer thickness of non-brownian hard-sphere suspensions in rectangular channel flows. Experiments in Fluids 57:1-15

Lauga E and Stone HA (2003) Effective slip in pressure-driven stokes flow. Journal of Fluid Mechanics 489:55-77

Lyon MK and Leal LG (1998a) An experimental study of the motion of concentrated suspensions in twodimensional channel flow. part 1. monodisperse systems. Journal of Fluid Mechanics 363:25-56

Lyon MK and Leal LG (1998b) An experimental study of the motion of concentrated suspensions in twodimensional channel flow. part 2. bidisperse systems. Journal of Fluid Mechanics 363:57-77

Medhi BJ, Kumar AA, and Singh A (2011) Apparent wall slip velocity measurements in free surface flow of concentrated suspensions. International Journal of Multiphase Flow 37:609-619

Sciacchitano A and Wieneke B (2016) Piv uncertainty propagation. Measurement Science and Technology 27:084006 